\title{
1 Programmed cell death as a black queen in microbial communities
}

2 Andrew Ndhlovu ${ }^{1}$, Pierre M. Durand ${ }^{2}$ and Grant Ramsey ${ }^{3}$

$3 \quad{ }^{1}$ Evolutionary Genomics Group, Department of Botany and Zoology, University of

$4 \quad$ Stellenbosch, Private Bag X1, Matieland 7602, South Africa

$5 \quad{ }^{2}$ Evolutionary Studies Institute, University of the Witwatersrand, Johannesburg, South Africa

$6 \quad$ Institute of Philosophy, KU Leuven, Leuven, Belgium

8 Email addresses

$9 \quad$ AN: andhlovu@sun.ac.za

10 PMD: pierre.durand@wits.ac.za

11 GR: grant@theramseylab.org

$12 @$ corresponding author

\begin{abstract}
Programmed cell death (PCD) in unicellular organisms is in some instances an altruistic trait. When the beneficiaries are clones or close kin, kin selection theory may be used to explain the evolution of the trait, and when the trait evolves in groups of distantly related individuals, group or multilevel selection theory is invoked. In mixed microbial communities, the benefits are also available to unrelated taxa. But the evolutionary ecology of PCD in communities is poorly understood. Few hypotheses have been offered concerning the community role of PCD despite its far-reaching effects. The hypothesis we consider here is that PCD is a black queen. The Black Queen Hypothesis $(\mathrm{BQH})$ outlines how public goods arising from a leaky function are exploited by other taxa in the community. Black Queen (BQ) traits are essential for community survival, but only some members bear the cost of possessing them, while others lose the trait. In addition, BQ traits have been defined in terms of adaptive gene loss, and it is unknown whether this has occurred for PCD. Our conclusion is that PCD fulfills the two most important criteria of a BQ (leakiness and costliness), but that more empirical data are needed for assessing the remaining two criteria. In addition, we hold that for viewing $\mathrm{PCD}$ as a BQ, the original BQH needs to include social traits. Thus, despite some empirical and conceptual shortcomings, the $\mathrm{BQH}$ provides a helpful avenue for investigating PCD in microbial communities.
\end{abstract}




\section{Introduction}

Unicellular organisms routinely undergo diverse forms of passive death, the causes of which include physical damage, starvation, irradiation, poison, and viral attack. In addition to these incidental forms of death, they also undergo active death. The active form of death-labelled programmed cell death (PCD) — has been observed in all the major bacterial and unicellular eukaryote crown groups (reviewed in Ameisen 2002; Rice and Bayles 2003; Franklin et al. 2006; Deponte 2008; Pérez Martín 2008; Kaczanowski et al. 2011; Nedelcu et al. 2011; Bayles 2014; Kasuba et al. 2015; Durand et al. 2016; Bidle 2016), and it is now clear that PCD has major implications in microbial communities (we define a microbial community as a population of microscopic taxa from different lineages that share space and resources, and which interact with each other in ways that impact their life history strategies). Questions concerning the impact of PCD on the community were explicitly raised more than a decade ago (Franklin et al. 2006). Although it is known that PCD contributes to the complexity of the community and plays a general role in evolutionary transitions (Durand et al. 2019) — such as the evolution of the eukaryote cell (Blackstone and Green 1999; Nedelcu and Michod 2003), multicellularity (Koonin and Aravind 2002; Michod 2003; Michod and Nedelcu 2003; Iranzo et al. 2014), and eusociality (Ronai et al. 2016) — a broad understanding of the ecological function of PCD in microbial communities is missing.

The central question that has occupied most evolutionary research in unicellular PCD is how natural selection could have selected for a trait that results in the elimination of the individual expressing it. To address this question, the focus has been on the levels and units of selection (Durand 2020). The ecological effects of PCD in microbial communities have received less attention and are poorly understood. Tinbergen's four questions concerning the mechanism, function, evolutionary history, and development are useful tools for examining the proximate and ultimate causes and the ecological relevance of any particular trait 

without permission. Instead, use the published version: https://doi.org/10.1111/mec.15757

(Tinbergen 1963; Bateson and Laland 2013). For the PCD trait, mechanism and function are particularly relevant to explain the multiple effects in microbial communities. We use the Berman-Frank mechanistic definition that PCD is an "active, genetically controlled, cellular self-destruction driven by a series of complex biochemical events and specialized cellular machinery" (Berman-Frank et al. 2004). The evolutionary history and development questions are less central here, although it is worth mentioning that depending on the ecological context, PCD can be adaptive for kin, groups, or populations (van Zandbergen et al. 2006; Durand et al. 2011, 2014; Refardt et al. 2013; Iranzo et al. 2014; Durand and Ramsey 2019; Vostinar et al. 2019), or it can be non-adaptive (Jiménez et al. 2009; Nedelcu et al. 2011; Proto et al. 2013; Ramisetty et al. 2015). The reader is referred elsewhere for further reading and evolutionary definitions of PCD (Reece et al. 2011; Berges and Choi 2014; Ramisetty et al. 2015; Durand and Ramsey 2019; Durand 2020).

Photosynthetic eukaryotic and prokaryotic microorganisms in aquatic environments have most frequently been used to examine PCD in microbial communities. When reports of programmed death in phytoplankton began to emerge, it quickly became apparent that PCD has major implications for the ecology of microbial communities (Franklin et al. 2006). This realization led to the proposal of a number of hypotheses to explain the evolutionary ecology of PCD in microbes (Frade and Michaelidis 1997; Blackstone and Green 1999; Segovia et al. 2003; Ameisen 2004; Kaczanowski et al. 2011; Nedelcu et al. 2011; Pepper et al. 2013; Iranzo et al. 2014; Klim et al. 2018). Phytoplankton contribute about $40 \%$ of global primary production (Field 1998; Geider et al. 2001) and their modes of death have far reaching biogeochemical effects (Bidle 2016). PCD impacts an organism's life history evolution in the microbial loop (Orellana et al. 2013), carbon export into the deep sea (Bidle 2016), resistance against viruses (Vardi et al. 2009), and population dynamics (Vardi et al. 1999). PCD is also implicated in the production of the transparent exopolymer polysaccharide (TEP) an 

without permission. Instead, use the published version: https://doi.org/10.1111/mec.15757

important component of the "sea skin" (Abada and Segev 2018; van Niekerk and Ndhlovu

2019). Furthermore, ecologically significant taxa including the nitrogen fixing

cyanobacterium Trichodesmium (Berman-Frank et al. 2004; Spungin et al. 2019), and the most abundant microbes in the global ocean-like Synechococcus (Thornton and Chen 2017) - undergo PCD. These organisms form massive blooms that collapse after several days or weeks, and their death contributes to the flow of nutrients in biogeochemical cycles (reviewed in Bidle 2016). However, the significance of PCD in these processes requires further studies.

The data from marine phytoplankton-prokaryote and other microbial communities indicates that PCD leads to a structuring of interactions between taxa. While there are several hypotheses put forward to explain the microbial ecology of PCD (Segovia et al. 2003; Ameisen 2004; Kaczanowski et al. 2011; Nedelcu et al. 2011; Pepper et al. 2013; Iranzo et al. 2014; Klim et al. 2018) there have been very few that explicitly examine the effect that PCD has in structuring microbial interactions and functional dependencies. One potential line of enquiry that may shed light on PCD in microbial communities is the Black Queen Hypothesis (BQH). The BQH, introduced by Morris et al. (2012), seeks to describe the conditions under which dependencies evolve in the microbial world. In this article, we ask whether PCD falls into the category of a black queen (BQ), since this would be of value for future studies. We evaluate each of the criteria imposed by the $\mathrm{BQH}$ and determine whether PCD fulfils these.

\section{The Black Queen Hypothesis}

The BQH is named after the black queen (the queen of spades) in the game of hearts (Morris et al. 2012), where the main strategy is to accumulate as few points as possible. The black queen is worth the most and is thus to be avoided. But someone is inevitably stuck with the queen of spades. A biological analogy can be made for multi-species microbial communities in which there are functions performed by members of the community that are vital to the 

without permission. Instead, use the published version: https://doi.org/10.1111/mec.15757

community but costly to the survival of the individuals performing the function (Figure 1).

No player of hearts wants the BQ (except in the case of shooting the moon-more on that later), but it is necessary that one person bears it. Similarly, there are microbial products that are costly and necessary for the community to produce, but that not every member of the community needs to produce. The example used by Morris et al. is the production of catalaseperoxidase (katG), a large, Fe-dependent enzyme that is the primary defense against external hydrogen peroxide $(\mathrm{HOOH})$ in cyanobacteria (Tichy and Vermaas 1999; Perelman et al. 2003). When viewed in isolation, it is puzzling that the ability to produce such a crucial enzyme would be lost. But these bacteria do not occur in isolation - they live as part of a community.

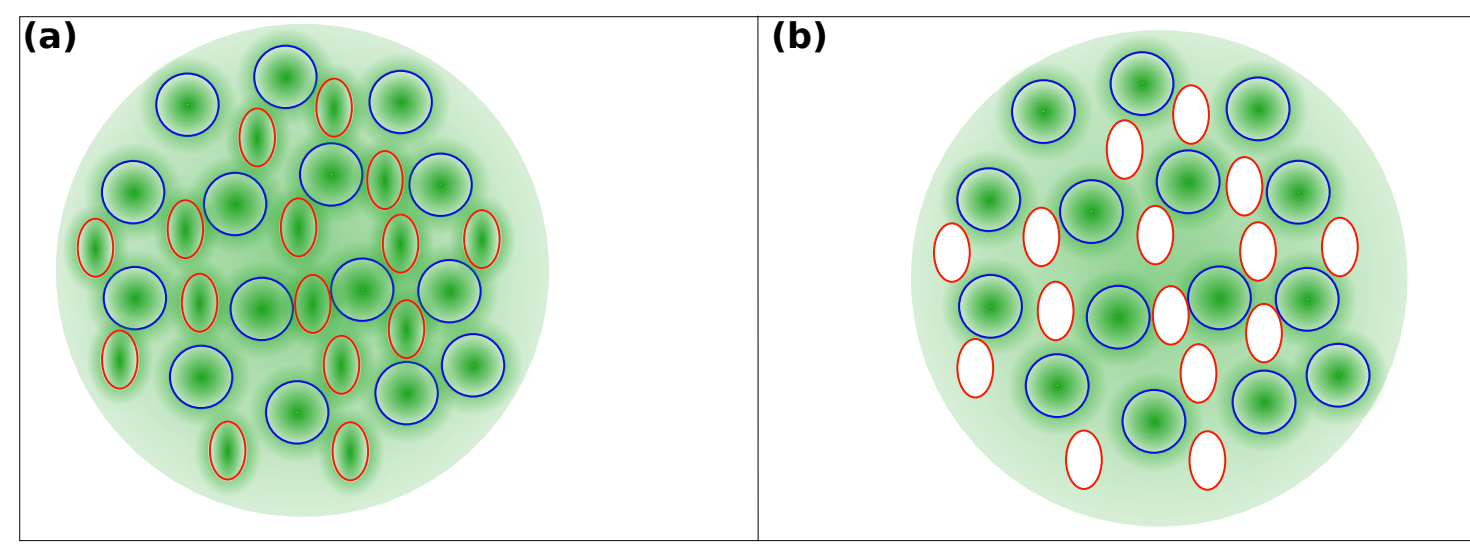

Figure 1. The BQH describes how the availability of public goods due to leakiness provides conditions for some taxon to relinquish the cost of performing a vital function. (a) Two taxon perform a leaky vital function, resulting in public goods. (b) These conditions lead to the red ellipsoid taxon relinquishing this costly activity becoming 'beneficiary' of the public goods provided by the blue circular 'helper' taxon.

From the point of view of any individual cyanobacterium, what is important is that katG is being produced, not that it is being produced by itself. Morris (2015) identifies the 

without permission. Instead, use the published version: https://doi.org/10.1111/mec.15757

katG enzyme as being leaky, benefiting both the producers and others in the community. The production of katG is expensive, and if an individual can escape the metabolic costs associated with synthesizing the enzyme, it will gain a fitness advantage. Comparative genomic data reveal that the enzyme is present in most cyanobacteria. However, all of the sequenced genomes of the members of the Prochlorococcus genus, and some members of the Synechococcus genus, lack the $k a t G$ gene despite the phylogenetic evidence of shared ancestry. In other words, the kat $G$ gene was lost in some lineages. The production of katG, therefore, fulfils the criteria of being a BQ. Investing in its production incurs a fitness cost but, just as the black queen in the card game is always present, the production of katG cannot be dispensed with. At least one species in any community of Prochlorococcus, Synechococcus, or any other genus of cyanobacterium, must produce katG to detoxify oxygen free radicals. At the level of the community, it is predicted that some species may exhibit functional gene loss, relying on other taxa to supply the vital gene products to others. The BQH shares a similar name to Van Valen's (1973) Red Queen Hypothesis (RQH) of evolutionary arms races between interacting taxa, resulting in constant extinction rates. Both hypotheses provide explanatory frameworks for functional interactions. However, in contrast with the $\mathrm{RQH}$, the $\mathrm{BQH}$ proposes a race to the bottom. Instead of gaining functions in order to win an evolutionary arms race, winners are those who have been successful at losing a vital but costly function. Organisms that lose these costly functions become 'beneficiaries' of the 'helpers', which are the organisms that perform the leaky, but vital and costly function. Morris et al. (2012) first described the detoxification of lethal reactive oxygen $(\mathrm{HOOH})$ as a BQ function in cyanobacteria, but subsequently went on to test $\mathrm{BQH}$ predictions in a similar dynamic in Escherichia coli, where species with $\mathrm{HOOH}$ resistance and sensitivity were able to coexist (Morris et al. 2014). These findings provided empirical evidence that BQs may be widespread and that the BQH may be a powerful lens through 

without permission. Instead, use the published version: https://doi.org/10.1111/mec.15757

which to examine the ecology of microbial communities. This has been supported by further searches for BQs in other microbial communities (Ankrah et al. 2018; Mas et al. 2016; Cairns et al. 2018; Billet et al. 2019).

The BQH shares similar features with widely known theories in the fields of social sciences and economics on public goods including the 'free-rider problem' (Sweeney 1973) and the 'tragedy of commons' (Hardin 1968). However, as Morris (2015) argued, the BQH goes further than these theories to provide an explanatory framework for how dependencies between helpers and beneficiary evolve in microbial communities. In comparison to existing theories on public goods, the $\mathrm{BQH}$ is more suited to capture the dynamics of the public goods of PCD in microbial communities. As a general hypothesis, the $\mathrm{BQH}$ seeks to describe the conditions under which costly traits lead to the evolution of dependencies in microbial communities.

\section{Criteria for the Black Queen Hypothesis}

Morris et al. (2012) noted that in all their examples the BQ was (i) leaky enough for the resulting public goods to be used by other species; (ii) costly (energetically or nutritionally expensive or bearing some other fitness cost); (iii) vital to the community, not just the producer, and (iv) performed by only a fraction of the community. We will treat these as general criteria for the identification of a BQ. Using data drawn chiefly from phytoplankton and prokaryote interactions, we will assess each of these in turn. After assessing the criteria, we will consider potential objections to our assessment and discuss the explanatory power of the $\mathrm{BQH}$ with respect to the role of PCD in community ecology. 
Criterion 1: BQ functions are leaky enough for the resulting public goods to be used by

\section{other species}

Leakiness was identified as the single most important requirement for a BQ trait to evolve (Morris 2015; Mas et al. 2016). It determines how dependencies between helpers and beneficiaries in microbial communities are structured via the release of substances across the “leakiness spectrum" (Morris 2015; p. 478), with biological functions ranging from purely public goods to purely private goods. Functions tend to be leaky if products are membrane permeable, extracellular, long lived, and modify the environment. Whether or not the functions are leaky is thus largely determined by the physico-chemical properties of the products and substrates.

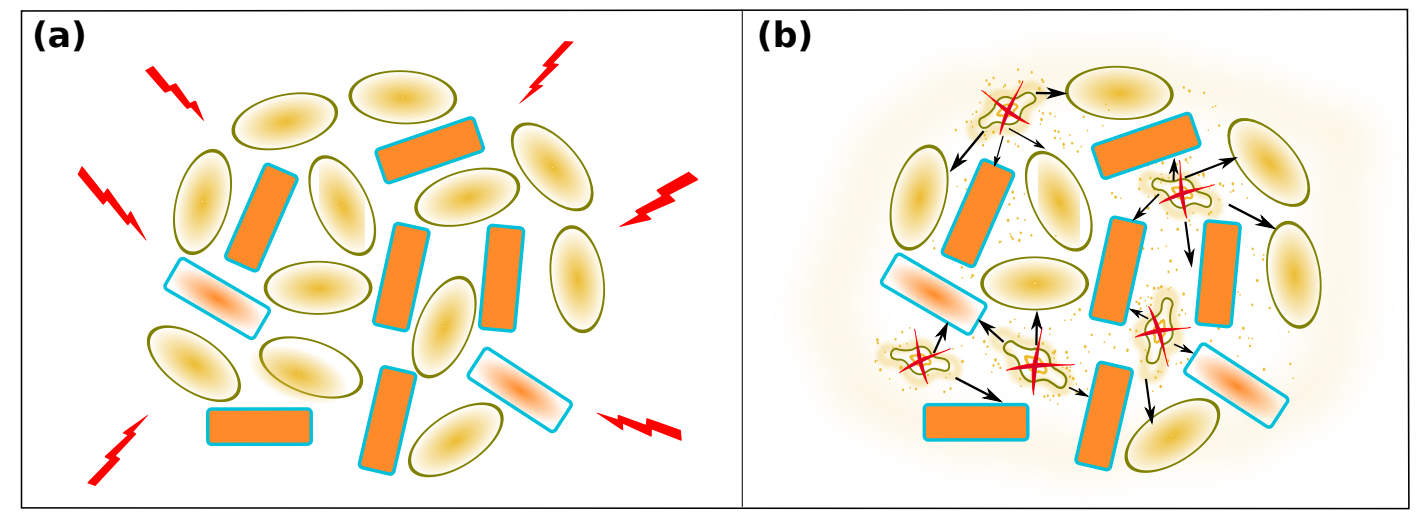

Figure 2. PCD is leaky, and public goods provide the conditions that may lead to the evolution of BQs. (a) Phytoplankton exist in microbial communities where they experience a variety of environmental stressors (lightining bolt). (b) Some members (ellipsoid) of the community undergo PCD. Cellular resources leak into the environment. These are available to other taxa (rectangular) leading to the evolution of dependencies.

Leakiness is an inevitable effect of PCD, since it results in molecules being extruded from the cell (Figure 2). The fitness effects of the substances released by cells dying by PCD have been examined in some instances. One demonstration of PCD leakiness and the effect of 

without permission. Instead, use the published version: https://doi.org/10.1111/mec.15757

the associated extruded molecules comes from the Great Salt Lake, Utah, USA (Orellana et al. 2013). In response to the onset of darkness, the halophilic chlorophyte Dunaliella salina undergoes PCD and releases dissolved organic matter (DOM) into the environment. Increases in growth rates of $D$. salina depended on the release of DOM during PCD. In addition, there was a mutual dependency discovered with a co-occurring prokaryote Halobacterium salinarum. The $H$. salinarum re-mineralized glycerol, one of the carbon sources present in the DOM released by dying $D$. salina cells (Orellana et al. 2013). In these and other situations, it is clear that PCD is a trait by which materials are released into the environment - and once released, can be used by others in the community, allowing functional dependencies to evolve. The 'leakiness' criterion is thus met.

\section{Criterion 2: A black queen is costly}

In Hearts, the player who ends up with the queen of spades (the BQ) is generally the loser, as this card is worth the same number of points as all the other cards combined. In the analogy with the card game, the BQ represents a function that is costly in terms of fitness. This is certainly the case for PCD. The substances released during PCD are nutritionally and energetically expensive. Chemical energy is used in the architectural restructuring of the cell and its organelles as revealed by ultrastructural studies (Arnoult et al. 2002; Moharikar et al. 2006; Jiménez et al. 2009; Durand et al. 2016), active biosynthesis and secretion of volatile organic compounds (Zuo et al. 2012) and lipid molecules (Sathe et al. 2019). While the resources released are costly to produce, if their release involves cell death —as is the case with PCD - then the trait clearly bears a significant cost.

\section{Criterion 3: A black queen is vital to the community}

What does it mean to be vital? In the classic BQ example (Morris et al. 2012), none of the individuals survive without the BQ, implying that the BQ is an obligate function. Clearly, 

without permission. Instead, use the published version: https://doi.org/10.1111/mec.15757

PCD and the leaky products hold no benefit for the dying cell, but the community benefits are manifested in two ways. First, there are benefits to conspecifics at the group or kin (inclusive fitness) level (Durand et al. 2011; Kaczanowski et al. 2011; Yordanova et al. 2013; Iranzo et al. 2014; Vostinar et al. 2019). Second, unrelated taxa in the community may also benefit, as seen in the Great Salt Lake microbial loop study (Orellana et al. 2013), which demonstrated that the production of glycerol during PCD is one of the nutrients driving the syntrophic interaction between $D$. salina and H. salinarum. Interactions like these play central roles in marine microbial communities in general (Bidle 2015). However, while PCD provides a fitness benefit in these situations, it is not clear whether it is always essential (at least in the short-term) for the survival of either the phytoplankton or the co-occurring prokaryotes in the community. In other instances, the PCD trait is indeed essential for survival. In Leishmania major infections, for example, PCD is essential for the survival of the parasite community in the host organism (van Zandbergen et al. 2006).

While the classic BQ example involved an essential function, it is not clear whether PCD is always vital for community survival. BQ products that are important but not vital to the community could be labelled 'weak BQs', whereas BQs producing vital—or nearly vital - traits are 'strong BQs'. It seems that most cases of PCD occupy the spectrum from weak BQ to strong BQ, but further investigation would be required to determine precisely where PCD falls on this spectrum.

\section{Criterion 4: Black queen functions are performed by only a fraction of the community}

The $\mathrm{BQH}$ asserts that it is an unstable equilibrium for all of the members of a community to produce a BQ trait. There is a selective advantage for the beneficiaries to dispense with the costs associated with the BQ. Due to the ubiquitous nature of the BQ products in the environment, there is a negative frequency dependency on fitness as organisms compete to 

without permission. Instead, use the published version: https://doi.org/10.1111/mec.15757

dispense with the costly function (Morris et al. 2012). Stability of the BQ is determined by the dynamics between helpers and beneficiaries.

Data from numerous model organisms indicate that under environmental conditions matching those in the field, only a proportion of cells undergo PCD (Vardi et al. 1999; Moharikar et al. 2006; van Zandbergen et al. 2010; Orellana et al. 2013; Bouderbala et al. 2018). If we can generalize from such studies, then Criterion 4 is satisfied. The difference with the prototypical BQ is that the fraction performing the BQ function is unrelated to the taxa who have lost the BQ. However, it seems that relatedness is not important for the evolution of BQs. The reason why others in the community do not undergo PCD has, however, not been explicitly studied. The $\mathrm{BQH}$ suggests that the function is lost because of its costly nature. On the other hand, as PCD-inducing factors vary with species (see for example, Nedelcu et al. 2011), this raises questions about whether non-PCD exhibiting taxa may simply have not yet been exposed to conditions that trigger their PCD.

\section{Adaptive gene loss and the evolution of dependencies in microbial communities}

Adaptive gene loss is not one of the criteria for BQs offered by Morris et al. (2012), but the examples they used all involve adaptive gene loss. Adaptive gene loss may well be an important mechanism underlying many cases of the loss of BQ traits, and there is ample evidence for the role of gene loss in evolution where resource conservation is the driving factor (Albalat and Canestro 2016). In the classic case of the KatG enzyme used by Morris et al. (2012), they used comparative genomics coupled with experimental evidence to identify the gene, its function, and to explain how the loss of the gene occurred as an adaptation.

In the case of PCD, the causal mechanism for the loss of function is unlikely to be as simple as a single gene loss. PCD is not a monogenic trait and in most instances the trait is manifested by complex polygenic machinery (Aravind et al. 1999; Uren et al. 2000; Durand and Coetzer 2008; Nedelcu 2009). PCD is also not a discrete all-or-nothing trait. It is a 

without permission. Instead, use the published version: https://doi.org/10.1111/mec.15757

continuous trait that is "probabilistic, branching, and non-discrete" (Durand and Ramsey 2019). It therefore seems unlikely that PCD can be lost through the loss of single genes, except in the simplest bacterial systems like E. coli (Refardt et al. 2013). However, if we accept the hypothesis for the ancient origins of PCD (Ameisen 2002), and that some taxa fail to show morphological markers of PCD in response to stress, we have to concede that the ability to undergo PCD may have been lost in some taxa. To our knowledge, however, this has not been demonstrated empirically or shown using genetic analyses.

Because loss of function (LoF) is not necessarily due to the loss of a gene or genenetwork, the $\mathrm{BQH}$ should not be based on adaptive gene loss. The one gene-one function concept is outdated and the loss of phenotypes can occur through changes in how genes are regulated (in the absence of their loss), but also by non-genetic means (Laland et al. 2015; Sultan 2015). These include processes like epigenetic inheritance, developmental bias, and phenotypic plasticity. In the Dunaliella-Halobacterium study, for example, approximately a third of the phytoplankton community underwent PCD (Orellana et al. 2013). In these organisms, the PCD trait is not monogenic (see Bidle 2015 for a broad outline of the mechanistic processes), though the reason for the other two-thirds not dying was not investigated. Of course, LoF mutations may have occurred, but we hold that the BQ classification is independent of whether it has occurred.

In the case of PCD, there is also an alternative to absolute LoF. Simply because the function appears to be lost, this does not mean that the inherited mechanism has actually been lost. The apparent disappearance of a trait may be part of microbial bet-hedging, something demonstrated by Libby et al. (2018), who argued that PCD is an accessory to microbial bet-hedging strategies that make use of stochastic phenotypic switching. Communities that face recurring but unpredictable environmental stresses may evolve such life-history strategies. The phenotype is manifested only under a particular set of 

without permission. Instead, use the published version: https://doi.org/10.1111/mec.15757

environmental conditions. In other words, there is a LoF, but it is facultative and the ability to produce the function is not eliminated from the organism's genome. Rather, the trait is plastic and switches on or off depending on the conditions.

One can therefore distinguish between two evolutionary responses to producing a BQ. One response is to lose the mechanisms capable of producing the $\mathrm{BQ}$, forcing other species to continue doing so. This has the advantage of being able to lose not just the function, but the machinery used to produce it. But there are costs. If the species lacking the ability finds itself surrounded by only other species also lacking this ability, then they will perish. The second response is to produce the resource facultatively, only when necessary if none of the other species present are producing it. The mechanistic properties of such a facultative BQ could depend on the type and function of the resource produced. Furthermore, it does not have to be constitutively expressed as in the case of the katG BQ dynamic, but could be environment dependent or triggered by stress as in the case of PCD. Furthermore, facultative production of a BQ requires sensing, and this ability can be more costly than constitutive traits. Members of such a species would produce the resource only if necessary, and only if no other species present are producing it. The never produce the $B Q$ and sometimes produce the $B Q$ are both strategies in the games of hearts played by microbial communities. Thus, neither pure LoF (as opposed to facultative LoF) nor gene loss are necessary for a trait to be a BQ. We concede that the empirical evidence to provide conclusive evidence of PCD as a BQ is currently lacking, and that future studies should be able to resolve the argument presented here.

\section{Morris's objection to sacrificial traits being BQs}

Morris (2015) claims that trait functions that require death or forgoing reproduction "have no private benefit for the producer and therefore are not BQ functions" (p. 476). This claim, however, is true only in the narrow sense, where selection is at the cell level and excludes inclusive fitness or selection at other levels. This seems overly restrictive, because evolution 

without permission. Instead, use the published version: https://doi.org/10.1111/mec.15757

by natural selection occurs at multiple levels of organization (Okasha 2006). The selection pressures in PCD also occur at multiple levels (Durand 2020). Because of this, it is important not to exclude these cases and limit selection to a particular level. Although there are no private benefits for the individual cell undergoing $\mathrm{PCD}$, there are fitness benefits arising at the kin/group level.

In cases where there is kin/group selection, the helper-beneficiary paradigm of the $\mathrm{BQH}$ is appropriate. It is now clear from several lineages that there are indeed kin- or grouplevel benefits arising from PCD (reviewed in Ameisen 2002; Berges and Choi 2014; Debrabant and Nakhasi 2003; Kaczanowski et al. 2011; Durand et al. 2016). In a multilevel selection context, there are private goods associated with PCD. The additional evidence that the benefits are selected for means that PCD in unicellular organisms can be defined as an altruistic adaptation to environmental stresses that lead to the death of the cell (Durand and Ramsey 2019).

However, there should be a distinction drawn between PCD as a nutritionally and energetically expensive trait and PCD as an altruistic trait. Morris et al. (2012) refer to a BQ as the former, but BQs should more generally be referred to as altruistic traits. Altruistic traits are by definition costly, but not all costly traits are altruistic. When the cell group is the target of selection, the suggestion that there are indeed private benefits for the producer is acceptable because the 'producer' is the group. This calls into question Morris's objection that sacrificial functions cannot be included in the $\mathrm{BQH}$. This is not to say that in all cases PCD should be viewed as a BQ. There are numerous proposed roles of PCD, and the trait may not always meet all of the necessary criteria to count as a BQ.

\section{Shooting the moon}

In the $\mathrm{BQH}$, avoidance of the $\mathrm{BQ}$ is the best strategy. Certainly, this is true for $\mathrm{PCD}$, since for the individual organism no death is better than death. But avoiding the $\mathrm{BQ}$, as a general 

without permission. Instead, use the published version: https://doi.org/10.1111/mec.15757

strategy, is beneficial only if other local species are stuck with the BQ. During the course of evolution, there may be times when no species bearing the BQ are to be found. Thus, while avoiding the BQ may be good in the short term, it may make the species more likely to go extinct. Producing the BQ may thus hold a benefit in the long term. This is indeed consistent with the bet-hedging argument made by Libby et al. (2018) referred to above.

Morris et al. (2012) note that being stuck with the black queen may not always be disadvantageous. In the card game there is a risky strategy to make the most of it-shooting the moon - that involves taking all the penalty cards. If successful, all the players except the holder of the black queen are penalized. The analogy with microorganisms is that at the community level, the taxon with the BQ trait may become a keystone species. Individuals with the BQ become ecologically essential for the survival of others in the community. Thus, one question to explore with $\mathrm{PCD}$ and the $\mathrm{BQH}$ is whether a benefit of exhibiting PCD is that it confers a large ecological importance on the species, thereby creating a stable niche.

Since the cell that exhibits PCD dies, the suggestion that a "shooting-the-moon" strategy is possible with PCD depends on PCD being selected for at the group or kin level. The question, therefore, is whether groups with PCD can outcompete other groups that do not exhibit the PCD trait. In an experiment to investigate PCD evolution, the costs and benefits of suicidal altruism in $E$. coli infected with an obligately lytic prophage were examined (Refardt et al. 2013). The authors found that altruistic suicide drove a population without PCD to extinction. Similarly, in experiments with L. major, groups of individuals without PCD were less fit than those with the PCD trait (van Zandbergen et al. 2010).

\section{Explanatory power of PCD being a BQ}

Multilevel selection models provide explanatory frameworks for how and why PCD could have evolved by natural selection (Reece et al. 2011; Refardt et al. 2013; Iranzo et al. 2014; 

without permission. Instead, use the published version: https://doi.org/10.1111/mec.15757

Durand 2020; Vostinar et al. 2019). What is unexplained, however, is the ecology of PCD in microbial communities comprising unrelated taxa. It is known that PCD has a significant effect on population structures, the partitioning of resources, and the evolution of costly functions (Franklin et al. 2006; Bidle 2016). The observation that PCD is unequally distributed across species in any particular community is unexplained and new avenues of enquiry are being sought. From our analyses, it seems that interpreting PCD as a BQ is promising as a potential explanation for the evolutionary dynamics of programmed forms of death in microbial communities. For example, the $\mathrm{BQH}$ predicts that dependencies between microbial communities will evolve when members of the community stop performing a vital and costly function. Seeing PCD as a BQ suggests that members of microbial communities in which all the species undergo PCD will likely not be an evolutionarily stable state. Species will be selected to liberate themselves from the BQ trait. Thus, the frequency of PCD in a community may carry important information about both the current function of the trait as well as its evolutionary history. For instance, a high frequency of PCD in a community may indicate that communities of this kind have a short evolutionary history, or that PCD is an exceptionally important feature of the community.

\section{Metacaspases: An example of the power of the BQH for investigating PCD}

Changes in the PCD pathway in the form of gene loss or LoF mutations represent the most logical place to find $\mathrm{BQ}$ functions and mechanistic explanations for the loss of PCD. While the principal proteins involved in the PCD machinery have largely been identified (Aravind et al. 1999), the molecular ecophysiology of PCD in unicellular organisms remains to be fully elucidated (Bidle 2015). In this section, we discuss proteins that are widely accepted to be executioners of the PCD pathway, and whose loss is likely to result in a loss of the PCD pathway. 
The emergence of PCD in unicellular eukaryotes has been attributed to the acquisition

of mitochondrial genes from an alpha-proteobacterium, a mitochondrion progenitor, coupled

with horizontal gene transfer events between the archaeo-eukaryote ancestor and bacteria

(Aravind et al. 1999; Koonin and Aravind 2002; Martijn and Ettema 2013). Caspases

(Cysteine-dependent ASPartyl-specific proteASE) are proteolytic cysteine specific proteases that have been identified to be at the heart of the PCD molecular machinery where they are and cell disintegration in metazoans (Cohen 1997; Thornberry and Lazebnik 1998).

Unicellular organisms lack caspases in their genomes, but distant homologs called metacaspases have been identified in a variety of unicellular taxa (Aravind et al. 1999; Uren et al. 2000; Tsiatsiani et al. 2011; Klemenčič and Funk 2019). Expression of metacaspase genes is increasingly being viewed as a proxy for PCD activity as numerous studies have correlated metacaspase expression with hallmarks of apoptotic-like PCD in a range of unicellular organisms (Kosec et al. 2006; Bidle et al. 2007; Bidle and Bender 2008; Tsiatsiani et al. 2011; Wang et al. 2017; Liu et al. 2018; Wang et al. 2018; Spungin et al. 2019). Although metacaspases have also been implicated in non-PCD related functions (Shrestha and Megeney 2012; Minina et al. 2017; Mata et al. 2019), they continue to be used to explore the origins and evolution of the PCD molecular machinery in unicellular organisms (Koonin and Aravind 2002; Choi and Berges 2013; Klemenčič and Funk 2019). Therefore, we propose that LoF of metacaspases genes would be a good indicator that the PCD pathway has been lost or compromised as set out in the $\mathrm{BQH}$.

Comparative analysis of prokaryote genomes reveal that there is variability in the number of metacaspases genes with members of the alphaproteobacteria, deltaproteobacteria, and cyanobacteria showing the greatest number of metacaspase genes (Asplund-Samuelsson et al. 2012). On the other hand, only a single metacaspase gene, $M C A 1$, has been identified in 

without permission. Instead, use the published version: https://doi.org/10.1111/mec.15757

the yeast Saccharomyces cerevisiae genome where the gene has been linked to apoptotic-like PCD (Madeo et al. 2002) and cytoprotection during aging (Hill and Nyström 2015). How the variability in the number of metacaspase genes between different taxa affects the PCD pathway remains unexplored. Evidence of the loss of metacaspase genes has been documented, coincidentally, in the same organisms which led to the formulation of the $\mathrm{BQH}$. Members of the Prochlorococcus and Synechococcus genera have been found to lack metacaspase genes in their genomes and are therefore suggested to be unable to undergo PCD (Bidle and Falkowski 2004; Asplund-Samuelsson et al. 2012). We are not aware of any work that has been carried out to investigate why these genes are missing. Future studies based on the lines of enquiry and theoretical frameworks discussed here will be able to assess whether loss of metacaspase genes are part of an evolutionary response to PCD functioning as a BQ in microbial communities.

\section{Concluding remarks}

Multilevel selection theory explains how unicellular species have evolved PCD by natural selection. What is much less clear is the ecological role of PCD in microbial communities. Despite the caveats and dearth of functional data from ecological studies, it does seem that PCD may be a BQ. PCD certainly is a leaky trait that is important for community survival and is quite obviously a costly trait. Whether there has been adaptive gene loss, however, is not clear, although the metacaspase example suggest that this may be the case. The hypothesis that PCD functions as a BQ may thus be a fruitful line of enquiry.

\section{Data availability statement}

Data sharing is not applicable to this article as no new data were created or analysed in this study. 


\section{$438 \quad$ Funding}

439 The support of the DST-NRF Centre of Excellence in Palaeosciences (CoE-Pal) toward this

440 research is hereby acknowledged. Opinions expressed and conclusions arrived at are those of

441 the authors and are not necessarily to be attributed to the CoE. 


\section{References}

Abada, A., and E. Segev. 2018. Multicellular Features of Phytoplankton. Frontiers in Marine Science 5.

Albalat, R., and C. Canestro. 2016. Evolution by gene loss. Nature Reviews Genetics 17:379391.

Ameisen, J. C. 2002. On the origin, evolution, and nature of programmed cell death: a timeline of four billion years. Cell Death and Differentiation 9:367-393. . 2004. Looking for death at the core of life in the light of evolution. Cell Death and Differentiation 11:4-10.

Ankrah, N. Y. D., B. Chouaia, and A. E. Douglas. 2018. The Cost of Metabolic Interactions in Symbioses between Insects and Bacteria with Reduced Genomes. (A. Wilson \& E. G. Ruby, eds.)mBio 9.

Aravind, L., V. M. Dixit, and E. V. Koonin. 1999. The domains of death: evolution of the apoptosis machinery. Trends Biochem Sci 24:47-53.

Arnoult, D., K. Akarid, A. Grodet, P. X. Petit, J. Estaquier, and J. C. Ameisen. 2002. On the evolution of programmed cell death: apoptosis of the unicellular eukaryote Leishmania major involves cysteine proteinase activation and mitochondrion permeabilization. Cell Death and Differentiation 9:65-81.

Asplund-Samuelsson, J., B. Bergman, and J. Larsson. 2012. Prokaryotic Caspase Homologs: Phylogenetic Patterns and Functional Characteristics Reveal Considerable Diversity. (A. Driks, ed.)PLoS ONE 7:e49888.

Bateson, P., and K. N. Laland. 2013. Tinbergen's four questions: an appreciation and an update. Trends in Ecology \& Evolution 28:712-718.

Bayles, K. W. 2014. Bacterial programmed cell death: making sense of a paradox. Nature Reviews Microbiology 12:63-9.

Berges, J. A., and C. J. Choi. 2014. Cell death in algae: physiological processes and relationships with stress. Perspectives in Phycology 1:103-112.

Berman-Frank, I., K. D. Bidle, L. Haramaty, and P. G. Falkowski. 2004. The demise of the marine cyanobacterium, Trichodesmium spp., via an autocatalyzed cell death pathway. Limnology and Oceanography 49:997-1005.

Bidle, K. D. 2015. The Molecular Ecophysiology of Programmed Cell Death in Marine Phytoplankton. Annual Review of Marine Science 7:341-375.

Bidle, K. D. 2016. Programmed Cell Death in Unicellular Phytoplankton. Current Biology 26:R594-607.

Bidle, K. D., and S. J. Bender. 2008. Iron Starvation and Culture Age Activate Metacaspases and Programmed Cell Death in the Marine Diatom Thalassiosira pseudonana. Eukaryotic Cell 7:223-236.

Bidle, K. D., and P. G. Falkowski. 2004. Cell death in planktonic, photosynthetic microorganisms. Nature Reviews Microbiology 2:643-655.

Bidle, K. D., L. Haramaty, E. R. J. Barcelos, and P. Falkowski. 2007. Viral activation and recruitment of metacaspases in the unicellular coccolithophore, Emiliania huxleyi. Proceedings of the National Academy of Sciences 104:6049-6054.

Billet, L., M. Devers, N. Rouard, F. Martin-Laurent, and A. Spor. 2019. Labour sharing promotes coexistence in atrazine degrading bacterial communities. Scientific Reports 9:18363.

Blackstone, N. W., and D. R. Green. 1999. The evolution of a mechanism of cell suicide. BioEssays 21:84-88.

Bouderbala, I., N. E1 Saadi, A. Bah, and P. Auger. 2018.. Acta Biotheoretica 66:257-278. 
Cairns, J., K. Koskinen, R. Penttinen, T. Patinen, A. Hartikainen, R. Jokela, L. Ruusulehto, et al. 2018. Black Queen Evolution and Trophic Interactions Determine Plasmid Survival after the Disruption of the Conjugation Network. mSystems 3.

Choi, C. J., and J. A. Berges. 2013. New types of metacaspases in phytoplankton reveal diverse origins of cell death proteases. Cell Death and Disease 4:e490-e490.

Cohen, G. M. 1997. Caspases: the executioners of apoptosis. Biochemical Journal 326:1-16.

Debrabant, A., and H. Nakhasi. 2003. Programmed cell death in trypanosomatids: is it an altruistic mechanism for survival of the fittest? Kinetoplastid Biol Dis 2:1-2.

Deponte, M. 2008. Programmed cell death in protists. Biochim Biophys Acta 1783:13961405.

Durand, P. M. 2020. The evolutionary origins of life and death. University of Chicago Press, Chicago, Illinois, USA.

Durand, P. M., M. M. Barreto Filho, and R. E. Michod. 2019. Cell Death in Evolutionary Transitions in Individuality. The Yale journal of biology and medicine 92:651-662.

Durand, P. M., R. Choudhury, A. Rashidi, and R. E. Michod. 2014. Programmed death in a unicellular organism has species-specific fitness effects. Biology Letters 10:20131088.

Durand, P. M., and T. L. Coetzer. 2008. Utility of computational methods to identify the apoptosis machinery in unicellular eukaryotes. Bioinform Biol Insights 2:101-17.

Durand, P. M., and G. Ramsey. 2019. The nature of programmed cell death. Biological Theory 14:30-41.

Durand, P. M., A. Rashidi, and R. E. Michod. 2011. How an organism dies affects the fitness of its neighbors. The American Naturalist 177:224-232.

Durand, P. M., S. Sym, and R. E. Michod. 2016. Programmed Cell Death and Complexity in Microbial Systems. Current Biology 26:R587-R593.

Field, C. B. 1998. Primary Production of the Biosphere: Integrating Terrestrial and Oceanic Components. Science 281:237-240.

Frade, J. M., and T. M. Michaelidis. 1997. Origin of eukaryotic programmed cell death: a consequence of aerobic metabolism? Bioessays 19:827-832.

Franklin, D. J., C. P. D. Brussaard, and J. A. Berges. 2006. What is the role and nature of programmed cell death in phytoplankton ecology? European Journal of Phycology 41:1-14.

Geider, R. J., E. H. Delucia, P. G. Falkowski, A. C. Finzi, J. P. Grime, J. Grace, T. M. Kana, et al. 2001. Primary productivity of planet earth: biological determinants and physical constraints in terrestrial and aquatic habitats. Global Change Biology 7:849-882.

Hardin, G. 1968. The tragedy of the commons. Science 162:1243-1248.

Hill, S. M., and T. Nyström. 2015. The dual role of a yeast metacaspase: What doesn't kill you makes you stronger. BioEssays 37:525-531.

Iranzo, J., A. E. Lobkovsky, Y. I. Wolf, and E. V. Koonin. 2014. Virus-host arms race at the joint origin of multicellularity and programmed cell death. Cell Cycle 13:3083-3088.

Jiménez, C., J. M. Capasso, C. L. Edelstein, C. J. Rivard, S. Lucia, S. Breusegem, T. Berl, et al. 2009. Different ways to die: cell death modes of the unicellular chlorophyte Dunaliella viridis exposed to various environmental stresses are mediated by the caspase-like activity DEVDase. Journal of Experimental Botany 60:815-828.

Kaczanowski, S., M. Sajid, and S. E. Reece. 2011. Evolution of apoptosis-like programmed cell death in unicellular protozoan parasites. Parasit Vectors 4:44.

Kasuba, K. C., S. L. Vavilala, and J. S. D’Souza. 2015. Apoptosis-like cell death in unicellular photosynthetic organisms - A review. Algal Research 12:126-133.

Klemenčič, M., and C. Funk. 2019. Evolution and structural diversity of metacaspases. Journal of Experimental Botany 70:2039-2047. 
Klim, J., A. Gładki, R. Kucharczyk, U. Zielenkiewicz, and S. Kaczanowski. 2018. Ancestral State Reconstruction of the Apoptosis Machinery in the Common Ancestor of Eukaryotes. G3: Genes, Genomes, Genetics 8:2121-2134.

Koonin, E. V., and L. Aravind. 2002. Origin and evolution of eukaryotic apoptosis: the bacterial connection. Cell Death and Differentiation 9:394-404.

Kosec, G., V. E. Alvarez, F. Agüero, D. Sánchez, M. Dolinar, B. Turk, V. Turk, et al. 2006. Metacaspases of Trypanosoma cruzi: Possible candidates for programmed cell death mediators. Molecular and Biochemical Parasitology 145:18-28.

Laland, K. N., T. Uller, M. W. Feldman, K. Sterelny, G. B. Müller, A. Moczek, E. Jablonka, et al. 2015. The extended evolutionary synthesis: its structure, assumptions and predictions. Proceedings of the Royal Society B: Biological Sciences 282:20151019.

Libby, E., W. W. Driscoll, and W. C. Ratcliff. 2018. Programmed cell death can increase the efficacy of microbial bet hedging. Scientific Reports 8:1120.

Liu, J., W. Cai, X. Fang, X. Wang, and G. Li. 2018. Virus-induced apoptosis and phosphorylation form of metacaspase in the marine coccolithophorid Emiliania huxleyi. Archives of Microbiology 200:413-422.

Madeo, F., E. Herker, C. Maldener, S. Wissing, S. Lächelt, M. Herlan, M. Fehr, et al. 2002. A caspase-related protease regulates apoptosis in yeast. Molecular Cell 9:911-917.

Martijn, J., and T. J. G. Ettema. 2013. From archaeon to eukaryote: the evolutionary dark ages of the eukaryotic cell. Biochemical Society Transactions 41:451-457.

Mas, A., S. Jamshidi, Y. Lagadeuc, D. Eveillard, and P. Vandenkoornhuyse. 2016. Beyond the black queen hypothesis. The ISME journal 10:2085-2091.

Mata, M. T., A. Palma, C. García-Gómez, M. López-Parages, V. Vázquez, I. Cheng-Sánchez, F. Sarabia, et al. 2019. Type II-metacaspases are involved in cell stress but not in cell death in the unicellular green alga Dunaliella tertiolecta. Microbial Cell 6:494-508.

Michod, R. 2003. Cooperation and Conflict Mediation during the Origin of Multicellularity. Hammerstein 2003.

Michod, R. E., and A. M. Nedelcu. 2003. Cooperation and conflict during the unicellularmulticellular and prokaryotic-eukaryotic transitions. Pages 195-208 in A. Moya and E. Font, eds. Evolution: from molecules to ecosystems. Oxford University Press, Oxford.

Minina, E. A., N. S. Coll, H. Tuominen, and P. V. Bozhkov. 2017. Metacaspases versus caspases in development and cell fate regulation. Cell Death and Differentiation 24:1314-1325.

Moharikar, S., J. S. D’Souza, A. B. Kulkarni, and B. J. Rao. 2006. Apoptotic-like cell death pathway is induced in unicellular chlorophyte Chlamydomonas reinhardtii (Chlorophyceae) cells following UV irradiation: detection and functional analyses1. Journal of Phycology 42:423-433.

Morris, J. J. 2015. Black Queen evolution: the role of leakiness in structuring microbial communities. Trends in Genetics 31:475-482.

Morris, J. J., R. E. Lenski, and E. R. Zinser. 2012. The Black Queen Hypothesis: evolution of dependencies through adaptive gene loss. mBio 3.

Morris, J. J., S. E. Papoulis, and R. E. Lenski. 2014. Coexistence of evolving bacteria stabilized by a shared black queen function: experimental evolution of a black queen community. Evolution 68:2960-2971.

Nedelcu, A. M. 2009. Comparative genomics of phylogenetically diverse unicellular eukaryotes provide new insights into the genetic basis for the evolution of the programmed cell death machinery. Journal of molecular evolution 68:256-268.

Nedelcu, A. M., W. W. Driscoll, P. M. Durand, M. D. Herron, and A. Rashidi. 2011. On the paradigm of altruistic suicide in the unicellular world. Evolution 65:3-20. 
Nedelcu, A. M., and R. E. Michod. 2003. Sex as a response to oxidative stress: the effect of antioxidants on sexual induction in a facultatively sexual lineage. Proc Biol Sci 270 Supp1 2:S136-9.

Okasha, S. 2006. Evolution and the levels of selection. Oxford University Press, Oxford.

Orellana, M. V., W. L. Pang, P. M. Durand, K. Whitehead, and N. S. Baliga. 2013. A Role for Programmed Cell Death in the Microbial Loop. (W.-C. Chin, ed.)PLoS ONE 8:e62595.

Pepper, J. W., D. E. Shelton, A. Rashidi, and P. M. Durand. 2013. Are internal deathpromoting mechanisms ever adaptive? Journal of Phylogenetics and Evolutionary Biology 1:113.

Perelman, A., A. Uzan, D. Hacohen, and R. Schwarz. 2003. Oxidative stress in Synechococcus sp. strain PCC 7942: various mechanisms for $\mathrm{H}_{2} \mathrm{O}_{2}$ detoxification with different physiological roles. Journal of Bacteriology 185:3654-60.

Pérez Martín, J. M. 2008. Programmed cell death in protozoa. Molecular biology intelligence unit. Springer-Verlag New York, New York, N.Y.

Proto, W. R., G. H. Coombs, and J. C. Mottram. 2013. Cell death in parasitic protozoa: regulated or incidental? Nat Rev Microbiol 11:58-66.

Ramisetty, B. C. M., B. Natarajan, and R. S. Santhosh. 2015. mazEF-mediated programmed cell death in bacteria: "What is this?" Critical Reviews in Microbiology 41:89-100.

Reece, S. E., L. C. Pollitt, N. Colegrave, and A. Gardner. 2011. The meaning of death: evolution and ecology of apoptosis in protozoan parasites. PLoS Pathogens 7:e1002320.

Refardt, D., T. Bergmiller, and R. Kümmerli. 2013. Altruism can evolve when relatedness is low: evidence from bacteria committing suicide upon phage infection. Proceedings of the Royal Society B: Biological Sciences 280:20123035.

Rice, K. C., and K. W. Bayles. 2003. Death's toolbox: examining the molecular components of bacterial programmed cell death. Molecular Microbiology 50:729-738.

Ronai, I., B. P. Oldroyd, and V. Vergoz. 2016. Queen pheromone regulates programmed cell death in the honey bee worker ovary: Queen pheromone regulates worker ovary cell death. Insect Molecular Biology 25:646-652.

Sathe, S., M. V. Orellana, N. S. Baliga, and P. M. Durand. 2019. Temporal and metabolic overlap between lipid accumulation and programmed cell death due to nitrogen starvation in the unicellular chlorophyte Chlamydomonas reinhardtii. Phycological Research 1-11.

Segovia, M., L. Haramaty, J. A. Berges, and P. G. Falkowski. 2003. Cell death in the unicellular chlorophyte Dunaliella tertiolecta. A hypothesis on the evolution of apoptosis in higher plants and metazoans. Plant Physiology 132:99-105.

Shrestha, A., and L. A. Megeney. 2012. The non-death role of metacaspase proteases. Frontiers in Oncology 2.

Spungin, D., K. D. Bidle, and I. Berman-Frank. 2019. Metacaspase involvement in programmed cell death of the marine cyanobacterium Trichodesmium. Environmental Microbiology 21:667-681.

Sultan, S. E. 2015. Organism and environment : ecological development, niche construction, and adaption (1st ed.). Oxford University Press.

Sweeney, J. W. 1973. An experimental investigation of the free-rider problem. Social Science Research 2:277-292.

Thornberry, N. A., and Y. Lazebnik. 1998. Caspases: Enemies Within. Science 281:13121316. 
Thornton, D. C. O., and J. Chen. 2017. Exopolymer production as a function of cell permeability and death in a diatom (Thalassiosira weissflogii) and a cyanobacterium (Synechococcus elongatus). (M. Cock, ed.)Journal of Phycology 53:245-260.

Tichy, M., and W. Vermaas. 1999. In vivo role of catalase-peroxidase in synechocystis sp. strain PCC 6803. Journal of Bacteriology 181:1875-82.

Tinbergen, N. 1963. On aims and methods of Ethology. Zeitschrift für Tierpsychologie 20:410-433.

Tsiatsiani, L., F. Van Breusegem, P. Gallois, A. Zavialov, E. Lam, and P. V. Bozhkov. 2011. Metacaspases. Cell Death and Differentiation 18:1279-1288.

Uren, A. G., K. O’Rourke, L. A. Aravind, M. T. Pisabarro, S. Seshagiri, E. V. Koonin, and V. M. Dixit. 2000. Identification of paracaspases and metacaspases: two ancient families of caspase-like proteins, one of which plays a key role in MALT lymphoma. Mol Cell 6:961-967.

van Niekerk, K., and A. Ndhlovu. 2019. Commentary: Multicellular Features of Phytoplankton. Frontiers in Marine Science 6.

Van Valen, L. 1973. A new evolutionary law. Evol Theory 1:1-30.

van Zandbergen, G., A. Bollinger, A. Wenzel, S. Kamhawi, R. Voll, M. Klinger, A. Müller, et al. 2006. Leishmania disease development depends on the presence of apoptotic promastigotes in the virulent inoculum. Proceedings of the National Academy of Sciences 103:13837-13842.

van Zandbergen, G., C. G. Luder, V. Heussler, and M. Duszenko. 2010. Programmed cell death in unicellular parasites: a prerequisite for sustained infection? Trends Parasitol 26:477-83.

Vardi, A., B. A. Van Mooy, H. F. Fredricks, K. J. Popendorf, J. E. Ossolinski, L. Haramaty, and K. D. Bidle. 2009. Viral glycosphingolipids induce lytic infection and cell death in marine phytoplankton. Science 326:861-865.

Vardi, B., I. Berman-Frank, T. Rozenberg, O. Hadas, A. Kaplan, and A. Levine. 1999. Programmed cell death of the dinoflagellate Peridinium gatunense is mediated by $\mathrm{CO}_{2}$ limitation and oxidative stress. Current Biology 9:1061-1064.

Vostinar, A. E., H. J. Goldsby, and C. Ofria. 2019. Suicidal selection: Programmed cell death can evolve in unicellular organisms due solely to kin selection. Ecology and Evolution 0:1-8.

Wang, H., T. Mi, Y. Zhen, X. Jing, Q. Liu, and Z. Yu. 2017. Metacaspases and programmed cell death in Skeletonema marinoi in response to silicate limitation. Journal of Plankton Research 39:729-743.

Wang, H., B. S. Park, W.-A. Lim, and J.-S. Ki. 2018. CpMCA, a novel metacaspase gene from the harmful dinoflagellate Cochlodinium polykrikoides and its expression during cell death. Gene 651:70-78.

Yordanova, Z. P., E. J. Woltering, V. M. Kapchina-Toteva, and E. T. Iakimova. 2013. Mastoparan-induced programmed cell death in the unicellular alga Chlamydomonas reinhardtii. Annals of Botany 111:191-205.

Zuo, Z., Y. Zhu, Y. Bai, and Y. Wang. 2012. Acetic acid-induced programmed cell death and release of volatile organic compounds in Chlamydomonas reinhardtii. Plant Physiol Biochem 51:175-84. 


\section{$684 \quad$ Figures}

685 Figure 1: The BQH describes how the availability of public goods due to leakiness 686 provides conditions for some taxa to relinquish the cost of performing a vital function. (a) 687 Two taxa perform a leaky vital function, resulting in public goods. (b) These conditions lead 688 to the red ellipsoid taxa relinquishing this costly activity becoming 'beneficiaries' of the 689 public goods provided by the blue circular 'helper' taxa.

690 Figure 2: PCD is leaky and public goods provide the conditions that may lead to 691 the evolution of BQs. (a) Phytoplankton exist in microbial communities where they 692 experience a variety of environmental stressors (lightning bolt). (b) Some members 693 (ellipsoid) of the community undergo PCD. Cellular resources leak into the environment. 694 These are available to other taxa (rectangular) leading to the evolution of dependencies. 